\title{
CONVOLUTION OPERATORS WITH TRIGONOMETRIC SPLINE KERNELS
}

\author{
by T. N. T. GOODMAN and S. L. LEE
}

(Received 1st December 1986)

\section{Introduction}

The Bernstein polynomials are algebraic polynomial approximation operators which possess shape preserving properties. These polynomial operators have been extended to spline approximation operators, the Bernstein-Schoenberg spline approximation operators, which are also shape preserving like the Bernstein polynomials [8].

The trigonometric counterpart of the Bernstein polynomials are the de la Vallee Poussin means. These are trigonometric polynomial approximation operators of the convolution type which are shape preserving [7]. Our objective is to study the properties of a class of convolution operators with trigonometric spline kernels, which are reminiscent of those of the de la Vallée Poussin means.

\section{Trigonometric B-splines}

Let $k$ be a positive integer, $h=2 \pi / k$ and $z_{v}=e^{i v h}, v=0,1, \ldots, k-1$, be the $k$ th roots of unity. Define on the unit circle $U$, the function

$$
M_{0}(z)= \begin{cases}1 & z \in \operatorname{arc}\left(z_{0}, z_{1}\right) \\ 0 & \text { otherwise }\end{cases}
$$

and for $n=0,1, \ldots, k-1$, define $M_{n}$ recursively by convolution, viz.

$$
M_{n}=M_{0}^{*} M_{n-1} \text {, }
$$

where the convolution * of two functions $f$ and $g$ on $U$ is defined by

$$
f^{*} g:=\int_{U} f\left(z \xi^{-1}\right) g(\xi) d \xi
$$

(see [6]).

If we denote the Fourier coefficients of a function $f$ by $\hat{f}_{v}, v \in \mathbb{Z}$, i.e.

$$
f_{v}=\int_{U} \frac{f(z)}{z^{v+1}} d z
$$


then

$$
\left(f^{*} g\right)_{v}=\hat{f}_{v} \hat{g}_{v-1}
$$

Now,

$$
\left(\hat{M}_{0}\right)_{v}= \begin{cases}2 \pi i / k & v=0 \\ \frac{1-e^{-i h v}}{v} & v \neq 0 .\end{cases}
$$

Hence

$$
\left(\hat{M}_{0}\right)_{v}=\left(\hat{M}_{0}\right)_{v}\left(\hat{M}_{0}\right)_{v-1} \ldots\left(\hat{M}_{0}\right)_{v-n}= \begin{cases}\frac{1}{k} \prod_{j=0}^{n}\left(\frac{1-e^{i(j-v) h}}{v-j}\right), & 0 \leqq v \leqq n \\ \frac{1}{2 \pi i} \prod_{j=0}^{n}\left(\frac{1-e^{i(n-v) h}}{v-j}\right), & \text { otherwise. }\end{cases}
$$

The notation $\Pi^{\prime}$ means that the factor corresponding to $j=v$ is 1 .

A straightforward computation gives

$$
\left(\hat{M}_{n}\right)_{v}=i^{n} e^{i(n+1)\left(\frac{1}{2} n-v\right) / 2} t_{v}
$$

where

$$
t_{v}= \begin{cases}\frac{2^{n}}{k} \prod_{j=0}^{n} \frac{\sin (v-j) h / 2}{(v-j)}, & 0 \leqq v \leqq n \\ \frac{2^{n}}{\pi} \prod_{j=0}^{n} \frac{\sin (v-j) h / 2}{(v-j)}, & \text { otherwise. }\end{cases}
$$

Hence, we can write

$$
M_{n}\left(e^{i x}\right)=i^{n} e^{i n x / 2} \sum_{v} t_{v} e^{i(v-n / 2)(x-(n+1) h / 2)}
$$

Since

$$
t_{v}=t_{n-v}, \quad v \in \mathbb{Z}
$$

it follows that the function

$$
T_{n}(x)=\sum_{v} t_{v} e^{i(v-n / 2)(x-(n+1) h / 2)}, \quad x \in[0,2 \pi)
$$


is a real function supported on the interval $(0,(n+1) h)$. It is called the trigonometric $B$ spline of degree $n$ with uniform knots at $v h, v=0,1, \ldots, n+1$. From (2.9) and (2.10), $T_{n}(x)$ is symmetrical about $(n+1) h / 2$ and we record the following relation from (2.8) and (2.10)

$$
T_{n}(x)=(-i)^{n} e^{-i n x / 2} M_{n}\left(e^{i x}\right), \quad x \in[0,2 \pi],
$$

and define $T_{n}$ to be $2 \pi$-periodic.

For the case where $n=2 m$ is an even integer, we define

$$
K_{m}(x):=T_{2 m}(x+(n+1) h / 2) / t_{m}, \quad x \in \mathbb{R} .
$$

Then

$$
K_{m}(x)=\sum_{v}\left(\hat{K}_{m}\right)_{v} e^{i v x}, \quad x \in \mathbb{R},
$$

where

$$
\begin{aligned}
\left(\hat{K}_{m}\right)_{v}= & t_{v+m} / t_{m} \\
& =\left\{\begin{array}{l}
\frac{(m !)^{2}(\sin (m-v) h / 2 \ldots \sin h / 2)(\sin (m+v) h / 2 \ldots \sin h / 2)}{(m-v) !(m+v) !(\sin h / 2 \ldots \sin m h / 2)^{2}},-m \leqq v \leqq m \\
\frac{k(m !)^{2} \sin (|v|-m) h / 2 \sin (|v|-m+1) h / 2 \ldots \sin (|v|+m) h / 2}{\pi(|v|-m) \ldots(|v|+m)(\sin h / 2 \ldots \sin m h / 2)^{2}}, \quad|v|>m .
\end{array}\right.
\end{aligned}
$$

Observe that $\left(\hat{K}_{m}\right)_{v}=0$ if and only if $|v|=p k-m, p k-m+1, \ldots, p k+m, p=1,2, \ldots$ In particular, if $k=2 m+1$, then $\left(\hat{K}_{m}\right)_{v}=0 \forall|v| \geqq m+1$, and

$$
\left(\widehat{K}_{m}\right)_{v}=\frac{(m !)^{2}}{(m-v) !(m+v) !}, \quad-m \leqq v \leqq m,
$$

so that

$$
K_{m}(x)=\omega_{m}(x):=\sum_{v=-m}^{m} \frac{(m !)^{2}}{(m-v) !(m+v) !} e^{i v x}, x \in \mathbb{R}
$$

are the de la Vallée Poussin kernels and

$$
V_{m}(f ; x):=\frac{1}{2 \pi} \int_{-\pi}^{\pi} \omega_{m}(x-t) f(t) d t, \quad x \in[0,2 \pi)
$$

are the de la Vallée Poussin means for a $2 \pi$-periodic integrable function $f$. It is wellknown that $V_{n}(f ;)$ converges uniformly to $f$ if $f$ is continuous. Furthermore the transform (2.15) is cyclic variation diminishing (see [6]). In particular, the kernel $\omega_{m}(t)$ is 
convex preserving, in the sense that if $\gamma(t)=\left(f_{1}(t), f_{2}(t)\right), t \in[0,2 \pi]$, where $f_{i}$ are $2 \pi$ periodic functions, is a convex curve in $\mathbb{R}^{2}$, then

$$
\Gamma(x):=\frac{1}{2 \pi} \int_{-\pi}^{\pi} \omega_{m}(x-t) f(t) d t \quad x \in[0,2 \pi]
$$

is also a convex curve in $\mathbb{R}^{2}$.

Our objective is to study the shape preserving and approximation properties of the convolution operators

$$
B_{m}(f ; x):=\frac{1}{2 \pi} \int_{-\pi}^{\pi} K_{m}(x-t) f(t) d t, \quad x \in[-\pi, \pi]
$$

From the above discussions on the Fourier coefficients $\left(\hat{K}_{m}\right)_{v}$ we see that the transform (2.17) cannot be cyclic variation diminishing because the condition $\left|\left(\hat{K}_{m}\right)_{v}\right| \geqq\left|\left(\hat{K}_{m}\right)_{v+1}\right|$, $v=0,1, \ldots$ is not satisfied (see [5]).

In Section 3 we study the general convolution kernel and give sufficient conditions for it to be "convex preserving". In Section 4, we show that the curve $\left(T_{n}^{\prime}(x), T_{n}(x)\right)$, $x \in[0,2 \pi]$ is a positively convex curve, and deduce that the kernel $T_{n}$ maps convex curves onto locally convex curves.

Section 5 deals with the approximation properties of the operator $B_{m}(f ; x)$. We show that for any continuous $2 \pi$-periodic function $f, B_{m}(f ; x)$ converges uniformly to $f(x)$ as $m \rightarrow \infty$. We also give an asymptotic estimate for $B_{m}(f ; x)-f(x)$ when $f$ is twice differentiable.

\section{Convexity preserving convolution kernels}

Let $K$ be a piecewise smooth, real $2 \pi$-periodic function and $\gamma(t)=\left(f_{1}(t), f_{2}(t)\right)$, $t \in[0,2 \pi]$, where $f_{1}(t)(i=1,2)$ are also piecewise smooth and $2 \pi$-periodic, be a closed curve in $\mathbb{R}^{2}$. We shall henceforward assume that all curves are piecewise smooth. Then

$$
\Gamma(x):=\int_{0}^{2 \pi} K(x-t) \gamma(t) d t, \quad x \in[0,2 \pi]
$$

is also a closed curve in $\mathbb{R}^{2}$. The kernel $K$ is said to be convex preserving if $\Gamma$ is convex whenever $\gamma$ is. By convexity of $\gamma$ we mean that it does not intersect any straight line more than twice. We also need the concept of local convexity. The curve $\Gamma(x)=\left(g_{1}(x)\right.$, $\left.g_{2}(x)\right), x \in[0,2 \pi]$ is locally convex if the Wronksian

$$
W\left(g_{1}^{\prime}, g_{2}^{\prime}\right):=\left|\begin{array}{ll}
g_{1}^{\prime}(x) & g_{2}^{\prime}(x) \\
g_{1}^{\prime \prime}(x) & g_{2}^{\prime \prime}(x)
\end{array}\right| \geqq 0 \quad \forall x \in[0,2 \pi]
$$

As usual, anticlockwise direction is taken as the positive orientation.

Theorem 3.1. A necessary and sufficient condition for the convolution transform (3.1) to 
map a positively convex curve onto a positively locally convex curve is that the kernel $\left(K^{\prime}(x), K(x)\right), 0 \leqq x \leqq 2 \pi$ be positively convex.

The necessity of the condition in Theorem 3.1 was proved in [7] by I. J. Schoenberg who attributed it to Loewner. The converse was recently established by Goodman and Lee [3].

\section{Shape preserving trigonometric $B$-spline kernel}

We shall consider convolution kernels $T_{n}, n=1,2, \ldots$, which are the trigonometric $B$ splines defined in Section 2. If $\gamma(t), t \in[0,2 \pi]$ is a closed curve, we define

$$
\Gamma_{n}(x)=\int_{0}^{2 \pi} T_{n}(x-t) \gamma(t) d t, \quad x \in[0,2 \pi]
$$

Our main result in this section is

Theorem 4.1. For $n=1,2, \ldots$, the curve $z_{n}(x)=\left(T_{n}^{\prime}(x), T_{n}(x)\right), x \in[0,2 \pi]$ is positively convex.

Since $z_{n}(x)=\left(T_{n}^{\prime}(x), T_{n}(x)\right), x \in[0,2 \pi]$ is positively convex, in view of Theorem 3.1 , we have the following

Corollary. The convolution transform (4.1) maps a positively convex curve onto a positively locally convex curve.

Remark. Theorem 4.1 is also true for real polynomial $B$-splines, the proof of which is much simpler.

We shall show that the curve $z_{n}(x)$ is positively locally convex by establishing inequality (4.12) by induction on $n$. That $z_{n}(x)$ is positively convex then follows easily from the symmetric, bell-shaped nature of $T_{n}(x)$ proved in Lemma 7 . The inductive step in the derivation of (4.12) requires a series of technical lemmas.

Lemma 1. For $n=1,2, \ldots$

$$
\begin{gathered}
n T_{n}(x)=2 \sin \frac{1}{2} x T_{n-1}(x)+2 \sin \frac{1}{2}((n+1) h-x) T_{n-1}(x-h) \\
T_{n}^{\prime}(x)=\cos \frac{1}{2} x T_{n-1}(x)-\cos \frac{1}{2}((n+1) h-x) T_{n-1}(x-h)
\end{gathered}
$$

The relations (4.2) and (4.3) are special cases of the recurrence relations for trigonometric $B$-splines and their derivatives (see [2], [9]). They can also be obtained directly and simultaneously by differentiating the convolution formula (2.4) via the relation (2.11).

Lemma. For $n=1,2, \ldots$

$$
(n-1) T_{n}^{\prime}(x)=2 \sin \frac{1}{2} x T_{n-1}^{\prime}(x)+2 \sin \frac{1}{2}((n+1) h-x) T_{n-1}^{\prime}(x-h) .
$$




$$
\begin{gathered}
T_{n}^{(2)}(x)+\frac{n}{4} T_{n}(x)=\cos \frac{1}{2} x T_{n-1}^{\prime}(x)-\cos \frac{1}{2}((n+1) h-x) T_{n-1}^{\prime}(x-h) \\
(n-2) T_{n}^{(2)}(x)-\frac{n}{4} T_{n}(x)=2 \sin \frac{1}{2} x T_{n-1}^{(2)}(x)+2 \sin \frac{1}{2}((n+1) h-x) T_{n-1}^{(2)}(x-h) \\
T_{n}^{(3)}(x)+\left(\frac{2 n-1}{4}\right) T_{n}^{\prime}(x)=\cos \frac{1}{2} x T_{n-1}^{(2)}(x)+\cos \frac{1}{2}((n+1) h-x) T_{n-1}^{(2)}(x-h) \\
(n-3) T_{n}^{(3)}(x)-\left(\frac{3 n+1}{4}\right) T_{n}^{\prime}(x)=2 \sin \frac{1}{2} x T_{n-1}^{(3)}(x)+2 \sin \frac{1}{2}((n+1) h-x) T_{n-1}^{(3)}(x-h)
\end{gathered}
$$

Proof of (4.4). Differentiating (4.2) gives

$$
\begin{aligned}
n T_{n}^{\prime}(x)= & \cos \frac{1}{2} x T_{n-1}(x)+\cos \frac{1}{2}((n+1) h-x) T_{n-1}(x-h) \\
& +2 \sin \frac{1}{2} x T_{n-1}^{\prime}(x)+2 \sin \frac{1}{2}((n+1) h-x) T_{n-1}^{\prime}(x-h) \\
= & T_{n}^{\prime}(x)+2 \sin \frac{1}{2} x T_{n-1}^{\prime}(x)+2 \sin \frac{1}{2}((n+1) h-x) T_{n-1}^{\prime}(x-h),
\end{aligned}
$$

and (4.4) follows. The other formulas are obtained in a similar manner.

Lemma 3. If $z_{n-1}(x)=\left(T_{n-1}^{\prime}(x), T_{n-1}(x)\right)$ is positively convex, then

$$
(n-1) T_{n}^{\prime}(x)^{2}-n T_{n}(x)\left(T_{n}^{(2)}(x)+\frac{n}{4} T_{n}(x)\right) \geqq 0, \quad 0 \leqq x \leqq 2 \pi .
$$

Proof. From (4.2), (4.3), (4.4) and (4.5),

$$
\begin{aligned}
(n-1) & T_{n}^{\prime}(x) T_{n}^{\prime}(x)-n T_{n}(x)\left(T_{n}^{(2)}(x)+\frac{n}{4} T_{n}(x)\right) \\
= & \sin x T_{n-1}(x) T_{n-1}^{\prime}(x)-\sin ((n+1) h-x) T_{n-1}(x-h) T_{n-1}^{\prime}(x-h) \\
& -2 \sin \frac{1}{2} x \cos \frac{1}{2}((n+1) h-x) T_{n-1}(x-h) T_{n-1}^{\prime}(x) \\
& +2 \cos \frac{1}{2} x \sin \frac{1}{2}((n+1) h-x) T_{n-1}^{\prime}(x-h) T_{n-1}(x) \\
& -\sin x T_{n-1}(x) T_{n-1}^{\prime}(x)+\sin ((n+1) h-x) T_{n-1}(x-h) T_{n-1}^{\prime}(x-h) \\
& +2 \sin \frac{1}{2} x \cos \frac{1}{2}((n+1) h-x) T_{n-1}(x) T_{n-1}^{\prime}(x-h) \\
& -2 \cos \frac{1}{2} x \sin \frac{1}{2}((n+1) h-x) T_{n-1}(x-h) T_{n-1}^{\prime}(x) \\
= & \sin \frac{1}{2}(n+1) h T_{n-1}^{\prime}(x-h) T_{n-1}(x)-\sin \frac{1}{2}(n+1) h T_{n-1}^{\prime}(x) T_{n-1}(x-h) \\
= & \sin \frac{1}{2}(n+1) h\left(T_{n-1}^{\prime}(x-h) T_{n-1}(x)-T_{n-1}^{\prime}(x) T_{n-1}(x-h)\right) \geqq 0
\end{aligned}
$$

because of the convexity of $\left(T_{n-1}^{\prime}(x), T_{n-1}(x)\right)$. 
Lemma 4. If $z_{n-1}(x)=\left(T_{n-1}^{\prime}(x), T_{n-1}(x)\right)$ is positively convex, then

$$
\left(n T_{n}(x) T_{n}^{(2)}(x)-(2 n-1) T_{n}^{\prime}(x)^{2}\right) \leqq 0 \quad 0 \leqq x \leqq 2 \pi .
$$

Proof. $n T_{n}(x) T_{n}^{(2)}(x)-(2 n-1) T_{n}^{\prime}(x)^{2}$

$$
\begin{aligned}
& =n T_{n}(x) T_{n}^{(2)}(x)-(n-1) T_{n}^{\prime}(x)^{2}+n^{2} T_{n}(x)^{2}-\left(n T_{n}^{\prime}(x)^{2}+\frac{n^{2}}{4} T_{n}(x)^{2}\right) \\
& =-\left\{(n-1) T_{n}^{\prime}(x)^{2}-n T(x)\left(\frac{n}{4} T_{n}(x)+T_{n}^{(2)}(x)\right)\right\}-\left(n T_{n}^{\prime}(x)^{2}+\frac{n^{2}}{4} T_{n}(x)^{2}\right)
\end{aligned}
$$

and the result follows by Lemma 3 .

The following lemma is geometrically obvious.

Lemma 5. If $z_{n-1}(x)=\left(T_{n-1}^{\prime}(x), T_{n-1}(x)\right)$ is positively convex, then

$$
T_{n-1}^{(2)}\left(x_{1}\right) T_{n-1}^{\prime}\left(x_{2}\right)-T_{n-1}^{(2)}\left(x_{2}\right) T_{n-1}^{\prime}\left(x_{1}\right) \geqq 0,
$$

for all $0 \leqq x_{1}<x_{2} \leqq 2 \pi$ for which the angle from the tangent $\left(T_{n-1}^{(2)}\left(x_{1}\right), T_{n-1}^{\prime}\left(x_{1}\right)\right)$ to $\left(T_{n-1}^{(2)}\left(x_{2}\right), T_{n-1}^{\prime}\left(x_{2}\right)\right)$ in the positive direction, does not exceed $180^{\circ}$.

Lemma 6. For $n=1,2, \ldots, \cos \frac{1}{2}(n+1) h+\frac{1}{2}(n-3) \leqq \frac{1}{2}(n-1) \cos \frac{1}{2} h$.

Proof. For $n=1,2, \ldots$,

$$
\begin{aligned}
\left(\frac{n-3}{2}\right)\left(\frac{h}{4}\right)^{2} & \leqq \frac{n(n+2)}{\pi^{2}}\left(\frac{h}{4}\right)^{2} \\
\Rightarrow\left(\frac{n-3}{2}\right) \sin ^{2} \frac{h}{4} & \leqq\left(\frac{n-3}{2}\right)\left(\frac{h}{4}\right)^{2} \\
& \leqq\left(\frac{n h}{4 \pi}\right)\left(\frac{(n+2) h}{4 \pi}\right) \\
& \leqq \sin \frac{1}{4} n h \sin \frac{1}{4}(n+2) h \\
\Rightarrow\left(\frac{n-3}{2}\right)\left(1-\cos \frac{1}{2} h\right) & \leqq \cos \frac{1}{2} h-\cos \frac{1}{2}(n+1) h \\
\Rightarrow \cos \frac{1}{2}(n+1) h+\frac{1}{2}(n-3) & \leqq \frac{1}{2}(n-1) \cos \frac{1}{2} h .
\end{aligned}
$$

Lemma 7. The function $T_{n}(x)$ is strictly increasing for $0 \leqq x \leqq(n+1) h / 2$ and strictly decreasing for $(n+1) h / 2 \leqq x \leqq(n+1) h$. 
Proof. The function $P_{n}(x)=T_{n}(x+(n+1) h / 2)$ is the central trigonometric $B$-spline supported on the interval $(-(n+1) h / 2,(n+1) h / 2)$ with knots at $(v-(n+1) / 2) h, v=0,1, \ldots n+1$. It is $C^{n-1}$, and its restriction on each interval $((v-(n+1) / 2) h$, $(v+1-(n+1) / 2) h)$ is a polynomial in $\sin ^{k} \frac{1}{2} x \cos ^{n-k} \frac{1}{2} x, k=0, \ldots, n$ (see [2]). By the transformation $t=\tan \frac{1}{2} x,-\pi<x<\pi$, we have

$$
\sin ^{k} \frac{1}{2} x \cos ^{n-k} \frac{1}{2} x=\tan ^{k} \frac{1}{2} x \cos ^{n} \frac{1}{2} x=t^{k} /\left(1+t^{2}\right)^{n / 2}
$$

(see [2]) and $P_{n}(x)=M_{n}(t) /\left(1+t^{2}\right)^{n / 2}, t \in \mathbb{R}$, where $M_{n}(t)$ is a real polynomial spline of degree $n$ with knots at $t_{v}=2 \arctan (v-(n+1) / 2) h, v=0,1, \ldots, n+1$, and supported at $\left(t_{0}, t_{n+1}\right)$. Hence $M_{n}(t)$ is a positive multiple of a polynomial $B$-spline of degree $n$.

If $-(n+1) h / 2<x_{1}<x_{2} \leqq 0$, then $t_{1}:=\tan \frac{1}{2} x_{1}<t_{2}:=\tan \frac{1}{2} x_{2} \leqq 0$, which implies that $M_{n}\left(t_{1}\right)<M_{n}\left(t_{2}\right)$, and $\left(1+t_{1}^{2}\right)^{n / 2}>\left(1+t_{2}^{2}\right)^{n / 2}$. It follows that $P_{n}(x)$ is strictly increasing for $-(n+1) h / 2 \leqq x \leqq 0$. Lemma 7 follows by symmetry.

Proof of Theorem 4.1. The proof will be by induction on $n$. The convexity of $z_{1}(x)$ $=\left(T_{1}^{\prime}(x), T_{1}(x)\right)$ and $z_{2}(x)=\left(T_{2}^{\prime}(x), T_{2}(x)\right)$ may be verified directly. Suppose $z_{1}(x)$ is positively convex for $i=1,2, \ldots, n-1$. We shall first show that

$$
W_{n}(x):=\left[T_{n}^{(2)}(x)\right]^{2}-T_{n}^{\prime}(x) T_{n}^{(3)}(x)>0 \quad \forall 0 \leqq x \leqq(n+1) h .
$$

By symmetry we need only to show that $W_{n}(x) \geqq 0$ for $0 \leqq x \leqq(n+1) h / 2$.

Differentiating equation (4.4), we obtain

$$
\begin{aligned}
(n-1) T_{n}^{(2)}(x)= & \cos \frac{1}{2} x T_{n-1}^{\prime}(x)-\cos \frac{1}{2}((n+1) h-x) T_{n-1}^{\prime}(x-h) \\
& +2 \sin \frac{1}{2} x T_{n-1}^{(2)}(x)+2 \sin \frac{1}{2}((n+1) h-x) T_{n-1}^{(2)}(x-h), \\
& 0 \leqq x \leqq 2 \pi .
\end{aligned}
$$

From (4.4), (4.5), (4.7) and (4.13), we have

$$
\begin{aligned}
(n-1) & W_{n}(x)+\frac{1}{4}(n-1)\left(n T_{n}(x) T_{n}^{(2)}(x)-(2 n-1)\left[T_{n}^{\prime}(x)\right]^{2}\right) \\
= & \left(\cos \frac{1}{2} x T_{n-1}^{\prime}(x)+\cos \frac{1}{2}((n+1) h-x) T_{n-1}^{\prime}(x-h)\right)^{2} \\
+ & 2 \sin \frac{1}{2}(n+1) h\left(T_{n-1}^{(2)}(x-h) T_{n-1}^{\prime}(x)-T_{n-1}^{(2)}(x) T_{n-1}^{\prime}(x-h)\right), \\
& 0 \leqq x \leqq 2 \pi .
\end{aligned}
$$

The second term on the left of equation (4.14) is non positive by Lemma 4 . The second term on the right of (4.14) is non negative for $0 \leqq x<n h / 2$ by Lemma 5 and Lemma 7 . Hence $W_{n}(x) \geqq 0$ for $0<x<n h / 2$. In order to complete the proof of (4.12), it remains to show that the inequality is also true for $n h / 2 \leqq x \leqq(n+1) h / 2$. To this end, let

$$
D_{n-1}(x):=T_{n-1}^{(2)}(x-h) T_{n-1}^{\prime}(x)-T_{n-1}^{(2)}(x) T_{n-1}^{\prime}(x-h) .
$$


Writing

$$
\begin{aligned}
D_{n-1}(x)= & \left(T_{n-1}^{(2)}(x-h)+\frac{n-1}{4} T_{n-1}(x-h)\right) T_{n-1}^{\prime}(x) \\
& \left.-\left(T_{n-1}^{(2)}(x)+\frac{n-1}{4} T_{n-1}(x)\right) T_{n-1}^{\prime}(x-h)\right\} \\
& -\left\{\frac{n-1}{4} T_{n-1}(x-h) T_{n-1}^{\prime}(x)-\frac{n-1}{4} T_{n-1}(x) T_{n-1}^{\prime}(x-h)\right\},
\end{aligned}
$$

and then substituting (4.3) and (4.5) for the first expression on the right and (4.2) and (4.3) for the second, with $n$ replaced by $n-1$, we obtain

$$
(n-2) D_{n-1}(x)=\left|\begin{array}{lll}
A_{n-1}(x) & B_{n-1}(x) & C_{n-1}(x) \\
T_{n-2}^{\prime}(x-2 h) & T_{n-2}^{\prime}(x-h) & T_{n-2}^{\prime}(x) \\
T_{n-2}(x-2 h) & T_{n-2}(x-h) & T_{n-2}(x)
\end{array}\right|,
$$

where

$$
\begin{gathered}
A_{n-1}(x):=(n-2) \cos \frac{1}{2} x \cos \frac{1}{2}(x-h)+\sin \frac{1}{2} x \sin \frac{1}{2}(x-h) \\
B_{n-1}(x):=\sin \frac{1}{2} x \sin \frac{1}{2}((n+1) h-x)-(n-2) \cos \frac{1}{2} x \cos \frac{1}{2}((n+1) h-x) \\
C_{n-1}(x):=(n-2) \cos \frac{1}{2}(n h-x) \cos \frac{1}{2}((n+1) h-x)+\sin \frac{1}{2}(n h-x) \sin \frac{1}{2}((n+1) h-x) .
\end{gathered}
$$

A straightforward computation yields

$$
\begin{gathered}
A_{n-1}(x)=\frac{1}{2}(n-3) \cos \frac{1}{2}\left(x-\frac{1}{2} h\right)+\frac{1}{2}(n-1) \cos \frac{1}{2} h \\
B_{n-1}(x)=\frac{1}{2}(n-1) \cos \frac{1}{2}(n+1) h+\frac{1}{2}(n-3) \cos \left(\frac{1}{2}(n+1) h-x\right),
\end{gathered}
$$

and

$$
C_{n-1}(x)=\frac{1}{2}(n-3) \cos \left(\frac{1}{2}(2 n+1) h-x\right)+\frac{1}{2}(n-1) \cos \frac{1}{2} h
$$

If $n h / 2 \leqq x \leqq(n+1) h / 2$, then

$$
\begin{gathered}
A_{n-1}(x) \geqq \frac{1}{2}(n-3) \cos \frac{1}{2} n h+\frac{1}{2}(n-1) \cos \frac{1}{2} h, \\
B_{n-1}(x) \leqq \frac{1}{2}(n-1) \cos \frac{1}{2}(n+1) h+\frac{1}{2}(n-3) \\
=\frac{1}{2}(n-3) \cos \frac{1}{2}(n+1) h+\cos \frac{1}{2}(n+1) h+\frac{1}{2}(n-3),
\end{gathered}
$$


and

$$
C_{n-1}(x) \geqq \frac{1}{2}(n-3) \cos \frac{1}{2}(n+1) h+\frac{1}{2}(n-1) \cos \frac{1}{2} h=: \alpha_{n} .
$$

It is easy to verify that $\alpha_{n}>0$ for $n \geqq 3$. Now, from (4.16) and (4.18), $A_{n-1}(x) \geqq \alpha_{n}$ and $C_{n-1}(x) \geqq \alpha_{n}$ for $n h / 2 \leqq x \leqq(n+1) h / 2$, and by Lemma 6 and (4.17), $B_{n-1}(x) \leqq \alpha_{n}$. It follows from (4.15) and the inductive hypothesis that for $n h / 2 \leqq x \leqq(n+1) h / 2$,

$$
(n-2) D_{n-1}(x) \geqq \alpha_{n}\left|\begin{array}{ccc}
1 & 1 & 1 \\
T_{n-2}^{\prime}(x-2 h) & T_{n-2}^{\prime}(x-h) & T_{n-2}^{\prime}(x) \\
T_{n-2}(x-2 h) & T_{n-2}(x-h) & T_{n-2}(x)
\end{array}\right| \geqq 0 .
$$

We can conclude by (4.14) and (4.19) that $W_{n}(x) \geqq 0$ for $n h / 2 \leqq x \leqq(n+1) h / 2$. Thus the relation (4.12) is established.

The inequality (4.12) means that the curve $z_{n}(x)=\left(T_{n}^{\prime}(x), T_{n}(x)\right), 0 \leqq x \leqq(n+1) h$, is positively locally convex. Also, $z_{n}(x)$ has no loops for $0<x<(n+1) h$. For if $z_{n}\left(x_{1}\right)=$ $z_{n}\left(x_{2}\right)$ for some $x_{1}, x_{2} \in(0,(n+1) h)$, then $T_{n}^{\prime}\left(x_{1}\right)=T_{n}^{\prime}\left(x_{2}\right)$ and $T_{n}\left(x_{1}\right)=T_{n}\left(x_{2}\right)$. The last equality implies $x_{1}=(n+1) h-x_{2}$, by symmetry, which means that $T_{n}^{\prime}\left(x_{1}\right)=-T_{n}^{\prime}\left(x_{2}\right)$. This is possible if and only if $x_{1}=0$ and $x_{2}=(n+1) h$ or $x_{1}=x_{1}=x_{2}=(n+1) h / 2$, by Lemma 7.

Since $z_{n}(x)$ is positively locally convex and has no loops for $0<x<(n+1) h$, then it must be positively convex.

\section{Approximation by trigonometric $B$-spline convolution operators}

The trigonometric $B$-spline convolution operators $B_{m}(f ; \cdot)$ defined by $(2.17)$ are bounded positive linear operators on the space of $2 \pi$-periodic continuous functions with the supremum norm. The kernels $K_{m}$ are even functions whose Fourier series representations may be expressed as

$$
K_{m}(x)=1+\sum_{v=1}^{\infty} 2\left(\hat{K}_{m}\right)_{v} \cos v x, \quad x \in \mathbb{R}
$$

where $\left(\hat{K}_{m}\right)_{v}, v=1,2, \ldots$, are given in (2.13). These operators fit into the rich theory of positive integral operators of convolution type (see [1], [4]). Here, we shall study only the convergence behaviour of $B_{m}(f ; \cdot)$.

The kernel $K_{m}$, in fact depends on two parameters $m$, the degree of the spline functions, and $h$ the size of the partition. Whenever we need to emphasize these parameters we shall write $K_{m}^{h} \equiv K_{m}$, and similarly $B_{m}^{h}=B_{m}$.

Theorem 5.1. For any continuous $2 \pi$-periodic function $f$,

$$
B_{m}^{h}(f ; \cdot) \rightarrow f \quad \text { uniformly on }[0,2 \pi] \text {, }
$$

if and only if $m \rightarrow \infty$. 
Proof. From (2.13), the first Fourier coefficient of the kernel $K_{m}^{h}$ is

$$
\left(\hat{K}_{m}^{h}\right)_{1}=\frac{m \sin (m+1) h / 2}{(m+1) \sin m h / 2}
$$

Clearly $\left(\hat{K}_{m}^{h}\right)_{1} \rightarrow 1$ if and only if $m \rightarrow \infty$. The result follows by Korovkin's Theorem [4].

Remark. The operators $B_{m}(f ; \cdot)$ do not converge to $f$ if the degree $m$ is fixed and the mesh size $h \rightarrow 0$. This is in contrast to the interpolating spline operators which converge to the interpolated function as the mesh size tends to zero.

Corollary. For $v=0,1, \ldots$,

$$
\left(\hat{K}_{m}^{h}\right)_{v} \rightarrow 1 \text { as } m \rightarrow \infty .
$$

Suppose $f^{(2)}(x)$ exists. If we write $f(t)=\phi\left(e^{i t}\right),-\pi \leqq t<\pi$, then we have

$$
\begin{aligned}
\phi\left(e^{i t}\right)= & \phi\left(e^{i x}\right)+\phi^{\prime}\left(e^{i x}\right)\left(e^{i t}-e^{i x}\right)+\frac{1}{2 !} \phi^{(2)}\left(e^{i x}\right)\left(e^{i t}-e^{i x}\right)^{2} \\
& +s\left(e^{i t}\right)\left(e^{i t}-e^{i x}\right)^{2}
\end{aligned}
$$

where $s$ is integrable and bounded, and

$$
\lim _{t \rightarrow x} s\left(e^{i t}\right)=0
$$

Applying the convolution operator to both sides of equation (5.5), with fixed $x$, gives

$$
\begin{aligned}
B_{m}(f ; x)= & f(x)+\phi^{\prime}\left(e^{i x}\right) e^{i x}\left(\left(\hat{K}_{m}^{h}\right)_{1}-1\right) \\
& +\frac{1}{2} \phi^{(2)}\left(e^{i x}\right) e^{i 2 x}\left(\left(\hat{K}_{m}^{h}\right)_{2}-2\left(\hat{K}_{m}^{h}\right)_{1}+1\right)+R_{m}(x)
\end{aligned}
$$

where the remainder term

$$
R_{m}(x)=\int_{-\pi}^{\pi} s\left(e^{i t}\right)\left(e^{i t}-e^{i x}\right)^{2} K_{m}(x-t) d t
$$

Since $f^{\prime}(x)=\phi^{\prime}\left(e^{i x}\right) i e^{i x}$ and $f^{(2)}(x)=-\phi^{\prime}\left(e^{i x}\right) e^{i x}-\phi^{(2)}\left(e^{i x}\right) e^{i 2 x}$, we can express (5.7) as

$$
B_{m}(f ; x)-f(x)=\left(1-\left(\hat{K}_{m}^{h}\right)_{1}\right) f^{(2)}(x)+\frac{1}{2} \phi^{(2)}\left(e^{i x}\right)\left\{\left(\hat{K}_{m}^{h}\right)_{2}-4\left(\hat{K}_{m}^{h}\right)_{1}+3\right\}+R_{m}(x) .
$$

Lemma 1. When $m \rightarrow \infty$ and $m h \rightarrow \alpha,(m+1)\left(1-\left(\hat{R}_{m}^{h}\right)_{1}\right) \rightarrow 1-\frac{1}{2} \alpha \cot \frac{1}{2} \alpha$.

Proof. A straightforward computation gives 


$$
\begin{aligned}
(m+1)\left(-\left(\hat{K}_{m}^{h}\right)_{1}\right. & =m+1-m \cos \frac{1}{2} h-m \cot \frac{1}{2} m h \sin \frac{1}{2} h \\
& =1+2 m \sin ^{2} \frac{1}{4} h-m \cot \frac{1}{2} m h \sin \frac{1}{2} h
\end{aligned}
$$

and the result follows on taking limit as $m \rightarrow \infty$ and $m h \rightarrow \alpha$.

Lemma 2. When $m \rightarrow \infty$ and $m h \rightarrow \alpha$,

$$
(m+1)\left\{\left(\hat{K}_{m}^{h}\right)_{2}-4\left(\hat{K}_{m}^{h}\right)_{1}+3\right\} \rightarrow 0 .
$$

\section{Proof. Writing}

$$
(m+1)\left\{\left(\hat{K}_{m}^{h}\right)_{2}-4\left(\hat{K}_{m}^{h}\right)_{1}+3\right\}=(m+1)\left(\left(\hat{K}_{m}^{h}\right)_{2}-\left(\hat{K}_{m}^{h}\right)_{1}\right)+3(m+1)\left(1-\left(K_{m}^{h}\right)\right),
$$

we see that the second term on the right of (5.11) converges to $3-\frac{3}{2} \alpha \cot \frac{1}{2} \alpha$, by Lemma 1 . A similar computation as in Lemma 1 shows that as $m \rightarrow \infty$ and $m h \rightarrow \alpha,(m+1)\left(\left(\hat{K}_{m}^{h}\right)_{2}-\right.$ $\left.\left(\widehat{K}_{m}^{h}\right)_{1}\right) \rightarrow \frac{3}{2} \cot \frac{1}{2} \alpha-3$, and we obtain (5.10).

Theorem 5.2. If $f^{(2)}(x)$ exists, then

$$
(m+1)\left\{B_{m}(f ; x)-f(x)\right\} \rightarrow\left(1-\frac{1}{2} \alpha \cot \frac{1}{2} \alpha\right) f^{(2)}(x) \text { as } m \rightarrow \infty \text { and } m h \rightarrow \alpha .
$$

Proof. Observe that from (5.9) and Lemmas 1 and 2, the theorem will be proved, if we show that

$$
(m+1) R_{m}(x) \rightarrow 0 \text { as } m \rightarrow \infty \text { and } m h \rightarrow \alpha
$$

For $\varepsilon>0$, choose $\delta>0$ such that $\left|s\left(e^{i(x-t)}\right)\right|<\varepsilon$ whenever $|t|<\delta$. Then from (5.8), we can write

$$
\begin{aligned}
(m+1) R_{m}(x) & =(m+1) \int_{-\pi}^{\pi} s\left(e^{i(x-t)}\right)\left(e^{i(x-t)}-e^{i x}\right)^{2} K_{m}(t) d t \\
& =I_{1}+I_{2}
\end{aligned}
$$

where

$$
I_{1}=(m+1) \int_{|t|<\delta} s\left(e^{i(x-t)}\right)\left(e^{i(x-t)}-e^{i x}\right)^{2} K_{m}(t) d t
$$

and

$$
I_{2}=(m+1) \int_{\delta \leqq|1| \leqq \pi} s\left(e^{i(x-t)}\right)\left(e^{i(x-t)}-e^{i x}\right)^{2} K_{m}(t) d t
$$


Now

$$
\begin{aligned}
\left|I_{1}\right| & \leqq(m+1) \varepsilon \int_{|\varepsilon|<\delta}\left|e^{-i t}-1\right|^{2} K_{m}(t) d t \\
& \leqq(m+1) \varepsilon \int_{-\pi}^{\pi} 2(1-\cos t) K_{m}(t) d t \\
& =2 \varepsilon(m+1)\left(1-\left(\hat{K}_{m}\right)_{1}\right) \\
& <4 \varepsilon \text { for sufficiently large } m,
\end{aligned}
$$

by Lemma 1. If $\left|s\left(e^{i t}\right)\right| \leqq M, t \in \mathbb{R}$,

$$
\begin{aligned}
\left|I_{2}\right| & \leqq(m+1) M \int_{\delta \leqq|t| \leqq \pi} 4 \sin ^{2} \frac{1}{2} t K_{m}(t) d t \\
& \leqq \frac{(m+1) M}{\delta^{2}} \int_{-\pi}^{\pi} 4 t^{2} \sin ^{2} \frac{1}{2} t K_{m}(t) d t \\
& \leqq \frac{(m+1) M \pi^{2}}{\delta^{2}} \int_{-\pi}^{\pi} 4 \sin ^{4} \frac{1}{2} t K_{m}(t) d t \\
& =\frac{(m+1) M \pi^{2}}{2 \delta^{2}} \int_{-\pi}^{\pi}(3+\cos 2 t-4 \cos t) K_{m}(t) d t \\
& =M \pi^{2}(m+1)\left\{\left(\hat{K}_{m}^{h}\right)_{2}-4\left(\hat{R}_{m}^{h}\right)_{1}+3\right\} / 2 \delta^{2}
\end{aligned}
$$

which tends to zero as $m \rightarrow \infty$ and $m h \rightarrow \alpha$. Combining (5.14)-(5.18), we obtain (5.13).

Recall that when $k=2 m+1, B_{m}(f ;)=V_{m}(f ;)$, the de la Vallée Poussin means of $f$, defined by (2.15). In this case $m h=m 2 \pi /(2 m+1) \rightarrow \pi$, as $m \rightarrow \infty$, and with $\alpha=\pi$, $\frac{1}{2} \alpha \cot \frac{1}{2} \alpha=0$. Theorem 5.2 then reduces to the following result of Natanson.

Corollary (I. P. Natanson). If $f^{(2)}(x)$ exists, then

$$
\lim _{m \rightarrow \infty}(m+1)\left\{V_{m}(f ; x)-f(x)\right\}=f^{(2)}(x) .
$$

\section{Kernels which are linear combinations of translates of $B$-splines}

Let $a_{j}^{k}, k=1,2, \ldots, j=0,1, \ldots, k-1$ be a triangular array of numbers and $K_{m}^{h}(x)$ be the normalised central trigonometric $B$-splines defined by (2.12), where $h=2 \pi / k$. Define

$$
S_{m}^{h}(x)=\sum_{j=0}^{k-1} a_{j}^{k} K_{m}^{h}(x-j h), \quad x \in[-\pi, \pi]
$$


and $S_{m}^{h}$ is $2 \pi$-periodic. The function $S_{m}^{h}$ is a trigonometric spline of degree $m$ with knots at $(v-(n+1) / 2) h, v \in \mathbb{Z}$ (For detail see [2]). The convolution operator

$$
\Omega_{m}^{h}(f ; x):=\frac{1}{2 \pi} \int_{-\pi}^{\pi} S_{m}^{h}(x-t) f(t) d t, \quad x \in[-\pi, \pi]
$$

is similar to the summation process for the Fourier series of $2 \pi$-periodic functions. We can write

$$
\Omega_{m}^{h}(f ; x)=\sum_{j=0}^{k-1} a_{j}^{k} \int_{-\pi}^{\pi} K_{m}^{h}(x-t-j h) f(t) d t
$$

Then

$$
\left|\Omega_{m}^{h}(f ; x)\right| \leqq\|f\| \sum_{j=0}^{k-1}\left|a_{j}^{k}\right| \quad \forall x \in[0,2 \pi]
$$

where \|\| is the supremum norm. Hence for any $m$ and $h, \Omega_{m}^{h}(f ;)$ is a bounded linear operator on the space $\tilde{C}([-\pi, \pi])$ of $2 \pi$-periodic continuous functions.

Theorem 6.1. Suppose that $\sum_{j=0}^{k-1}\left|a_{j}^{k}\right|, k=1,2, \ldots$, is bounded. Then for $f \in \widetilde{C}([-\pi \pi])$,

$$
\begin{gathered}
\Omega_{m}^{h}(f ;) \rightarrow f \text { uniformly on }[-\pi, \pi] \text { as } m \rightarrow \infty \text { if and only if } \\
\qquad \sum_{j=0}^{k-1} a_{j}^{k} \omega^{v j} \rightarrow 1 \text { as } k=2 \pi / h \rightarrow \infty
\end{gathered}
$$

where $\omega=e^{-i h}$.

Proof. Substituting $f(t)=e_{v}(t):=\exp (i v t), v \in \mathbb{Z}$ in (6.3), we have

$$
\Omega_{m}^{h}\left(e_{v} ; x\right)=e_{v}(x)\left(\hat{K}_{m}^{h}\right)_{v} \sum_{j=0}^{k-1} a_{j}^{k} \omega^{v j}
$$

It follows from (5.4) that

$$
\Omega_{m}^{h}\left(e_{v} ;\right) \rightarrow e_{v} \text { uniformly on }[-\pi \pi] \text {, as } m \rightarrow \infty
$$

if and only if (6.6) holds. The result then follows.

Acknowledgement. The second author, S. L. Lee, acknowledges with thanks the support of SERC, U.K. 


\section{REFERENCES}

1. P. L. Butzer and R. J. Nessel, Fourier Analysis and Approximation (Academic Press, 1971).

2. T. N. T. Goodman and S. L. Lee, $B$-splines on the circle and trigonometric $B$-splines, Proc. Conference on Approximation Theory and Spline Functions (S. P. Singh, J. W. H. Bury and B. Watson, eds., St. Johns, Newfoundland, Reidel Pub. Co., 1983), 297-325.

3. T. N. T. Goodman and S. L. LeE, Convexity preserving convolution operators, Proc. Conference on Constructive Approximation, Edmonton (1986), to appear.

4. P. P. Korovkin, Linear Operators and Approximation Theory (Hindustan Press, 1960).

5. J. Mairhuber, I. J. Schoenderg and R. Williamson, On variation diminishing transformations on the circle, Rend. Circ. Mat. Palermo (2) 8 (1959), 241-270.

6. I. J. Schoenberg, On polynomial splines on the circle I, Proc. Conference on Constructive Theory of Functions (Budapest, 1972), 403-433.

7. I. J. Schoenberg, On variation diminishing approximation methods, On Numerical Approximation (Ed. R. E. Langer, University of Wisconsin Press, 1959), 249-274.

8. I. J. Schoenderg, On spline functions, Inequalities (Ed. O. Shisha, Academic Press, 1967), 255-290.

9. L. L. Schumaker, Spline functions: Basic theory (Wiley, New York, 1981).

Department of Mathematical Sciences

ThE UNIVERSITY

DUNDEE DD1 4HN

SCOTLAND
School of Mathematical Sciences UNIVERSITY OF SCIENCES OF MALAYSIA Penang

MaLAYSIA 